\title{
Nostril Base Augmentation Effect of Alveolar Bone Graft
}

\author{
Woojin Lee, Hyung Joon Park, Hyun Gon Choi, Dong Hyeok Shin, Ki Il Uhm \\ Department of Plastic and Reconstructive Surgery, Konkuk University Medical Center, Konkuk University School of Medicine, Seoul, Korea
}

Background The aims of alveolar bone grafting are closure of the fistula, stabilization of the maxillary arch, support for the roots of the teeth adjacent to the cleft on each side. We observed nostril base augmentation in patients with alveolar clefts after alveolar bone grafting. The purpose of this study was to evaluate the nostril base augmentation effect of secondary alveolar bone grafting in patients with unilateral alveolar cleft.

Methods Records of 15 children with alveolar clefts who underwent secondary alveolar bone grafting with autogenous iliac cancellous bone between March of 2011 and May of 2012 were reviewed. Preoperative and postoperative worm's-eye view photographs and reconstructed three-dimensional computed tomography (CT) scans were used for photogrammetry. The depression of the nostril base and thickness of the philtrum on the cleft side were measured in comparison to the normal side. The depression of the cleft side pyriform aperture was measured in comparison to the normal side on reconstructed three-dimensional CT.

Results Significant changes were seen in the nostril base $(P=0.005)$, the philtrum length $(P=0.013)$, and the angle $(P=0.006)$. The $C T$ measurements showed significant changes in the pyriform aperture $(\mathrm{P}<0.001)$ and the angle $(\mathrm{P}<0.001)$.

Conclusions An alveolar bone graft not only fills the gap in the alveolar process but also augments the nostril base after surgery. In this study, only an alveolar bone graft was performed to prevent bias from other procedures. Nostril base augmentation can be achieved by performing alveolar bone grafts in children, in whom invasive methods are not advised.

Keywords Alveolar process / Cleft palate / Alveoloplasty / Bone transplantation

\author{
Correspondence: Ki II Uhm \\ Department of Plastic and \\ Reconstructive Surgery, \\ Konkuk University Medical Center, \\ Konkuk University School of Medicine, \\ 120-1 Neungdong-ro, Gwangjin-gu, \\ Seoul 143-729, Korea \\ Tel: $+82-2-2030-5235$ \\ Fax: +82-2-2030-5249 \\ E-mail: kiumps@hanmail.net
}

No potential conflict of interest relevant to this article was reported.

\section{INTRODUCTION}

Four purposes of alveolar bone grafting are closure of the fistula, stabilization of the maxillary arch, support for the roots of the teeth adjacent to the cleft on each side, and support for a prosthesis [1]. However, we believe that nostril base augmentation of the alveolar cleft is another important aim of the alveolar bone graft. We have used 4 different methods to augment the nostril base in adults. This augmentation process involves elevating the skeletal base by cortical bone grafting, bulking the muscle using a muscle suture, performing skin and muscle tissue augmentation, and extending the perpendicular length of the nostril base lining. These methods have been used for adult patients, but Alveolar bone grafting alone should be performed for patients with alveolar clefts at 8 and 9 years of age before the cuspid erupts into the cleft [2]. We predicted that alveolar bone grafting would augment the nostril base in these patients. 
Table 1. Patient characteristics $(n=15)$

\begin{tabular}{|ll|}
\hline Characteristic & Value \\
\hline Age (yr) & \\
Average (range) & $9.4(7-15)$ \\
Sex (male:female) & $9: 6$ \\
Cleft side (left:right) & $8: 7$ \\
\hline
\end{tabular}

\section{METHODS}

We retrospectively reviewed a prospectively maintained database of consecutive pediatric patients with unilateral cleft lip alveolus who underwent secondary alveolar bone grafting using autogenous iliac cancellous bone between March 2011 and May 2012. All of the operations were performed by the senior author (K.I.U.). Thirty patients with unilateral alveolar clefts underwent alveolar bone grafting during this period. Patients who did not have preoperative and/or 6-month postoperative photographs or three-dimensional (3D) computed tomography (CT) data were excluded from the study. Patients who were too old to be classified as a child, <18 years old, were excluded [3]. Fifteen patients were included in the final study. The mean age at the time of surgery was 9.4 years (Table 1 ). All of the patients were followed up for at least 6 months.

All of the subjects received a gingivoperiosteoplasty and oronasal fistula repair with the alveolar bone graft. To measure the amount of harvested cancellous bone, a 5-mL syringe was filled with the harvested bone and the plunger was pressed until no more squeezing was observed. A 4-0 absorbable multifilament suture was used to repair the gingivoperiosteum. Approximately $1 \mathrm{~cm}^{3}$ of bone was grafted into the cleft for alveolar cleft repair.

We obtained preoperative and 6-month postoperative worm'seye view photographs and facial CT data. The photography and CT data were measured using Photoshop CS4 (Adobe Systems Inc., San Jose, CA, USA). In the photographic measurements, the line connecting the lowest border of both earlobes was used as the horizontal line [4]. A line parallel to this was drawn on the lowest point of the normal nostril. From this line, the height of the depression ( $n$ ) and thickness of the philtrum ( $p$ ) was measured. The angle (a) was measured with the line connecting both nostril bases using the ruler tool (Figs. 1, 2). The value provided by Photoshop is not an absolute value; rather, it varies according to picture and screen resolution. However, the numerical value is stable with regard to forming a ratio when used within the same image. Therefore, the longest distance of the radius of the corneal limbus ( $r$ ) was measured to obtain the $n / r$ ratio. The $n / r$ ratio was used to represent the length of the depression. The worm's-eye view of the $3 \mathrm{D}$ reconstructed

\section{Fig. 1. Landmarks used in the photographic analysis}

$a$, angle of depression;
$n$, depression length;
$p$, thickness of the

philtrum.

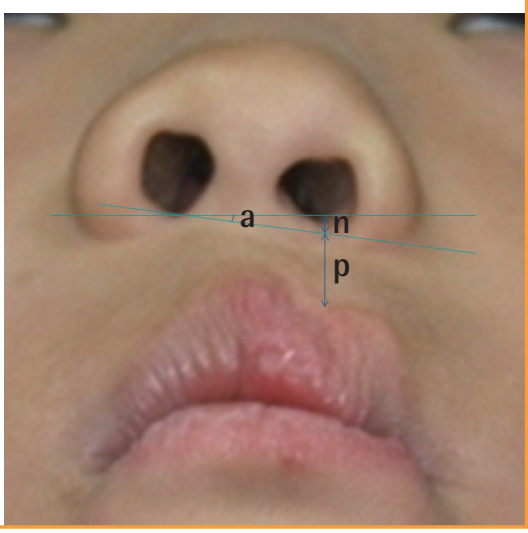

\section{Fig. 2. Diagram of the photographic analysis}

a, angle of depression;

$n$, depression length;

$p$, thickness of the philtrum.

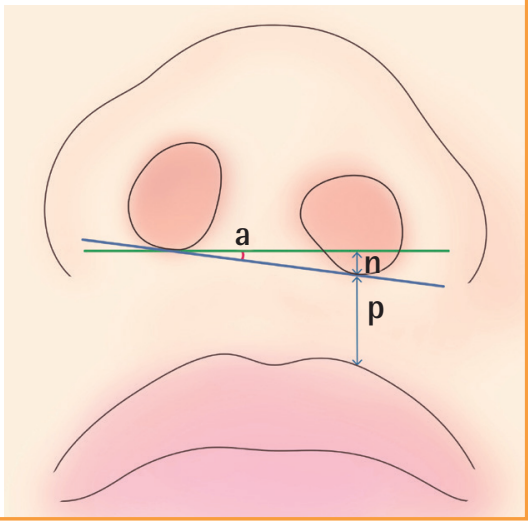

facial CT image was evaluated (Fig. 3). The line connecting the lowest point of the orbital rim was used as the horizontal line. A line parallel to this was drawn on the lowest point of the normal pyriform aperture. From this line, the $\mathrm{n}$ and a values were measured using the ruler tool. The $1-\mathrm{cm}$ ruler on the CT image was measured in pixels, and the $\mathrm{n}$ value was divided by this value to convert the pixel count into centimeters of depression length. We recorded these data and analyzed them using Excel 2007 (Microsoft Corp., Redmond, WA, USA).

The Wilcoxon signed-rank test was performed because the study included $<30$ patients. All statistical analysis was performed with SPSS ver. 17 (SPSS Inc., Chicago, IL, USA).

\section{RESULTS}

No postoperative surgical complications such as graft failure, wound dehiscence, flap necrosis, or infection were reported. A standardized photograph and 3D facial CT were taken at 6 months after the surgery.

In the analysis of the photographs, the nostril base height $(n / r)$ value showed a significant elevation from $0.00 \pm 0.12$ to $0.09 \pm$ $0.13(\mathrm{P}=0.005)$ and the $\mathrm{p} / \mathrm{r}$ value showed a significant change 
Fig. 3. Landmarks in three-dimensional computed tomography image

a, angle of depression; $n$, depression length.

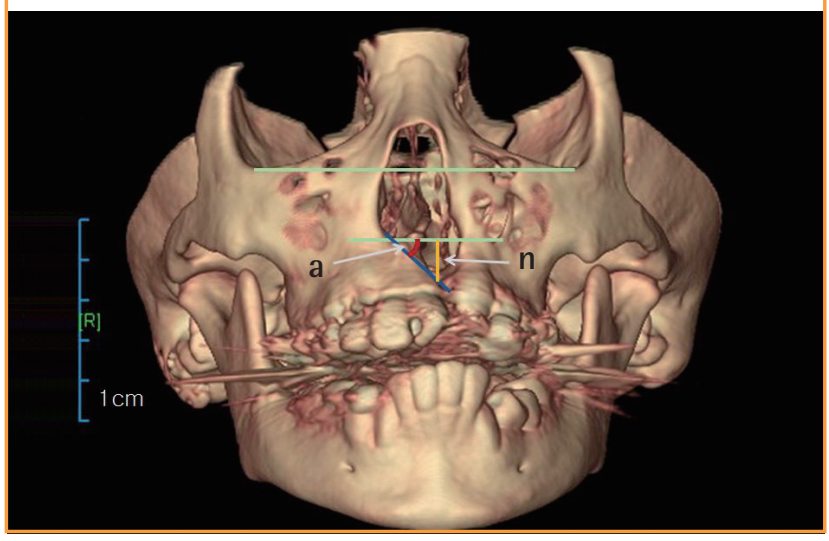

\section{Fig. 4. Worm's-eye view photographs}

(A) Preoperative photograph. (B) Photograph 6 months postoperatively.
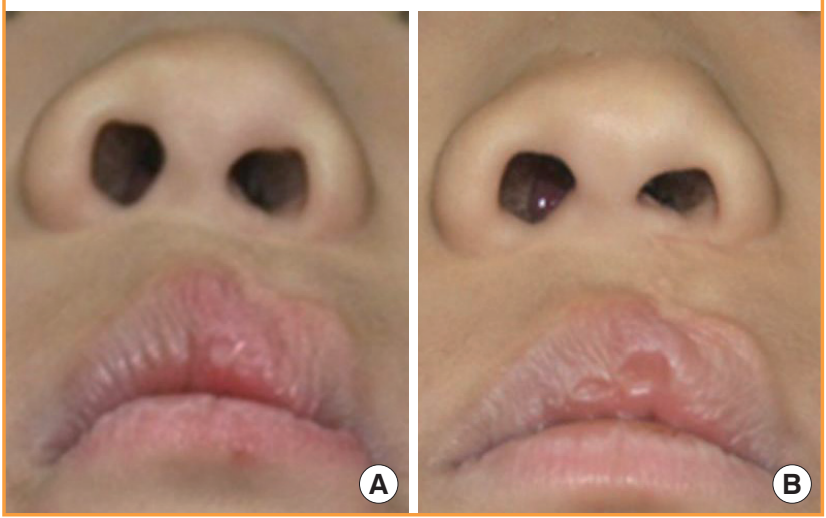

Fig. 5. Three-dimensional reconstructed facial computed tomography image

(A) Preoperative three-dimensional computed tomography. (B) Three-dimensional computed tomography 6 months postoperatively.
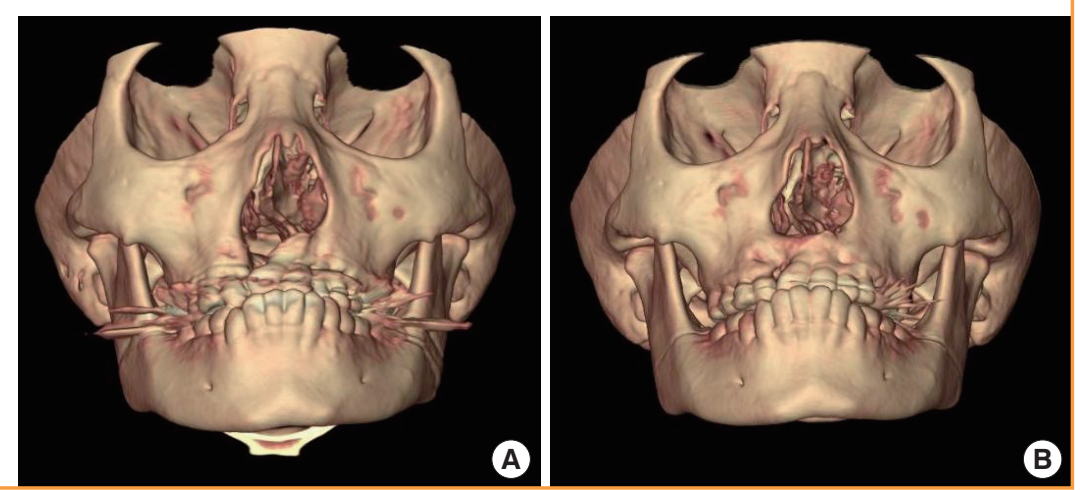

from $0.69 \pm 0.25$ to $0.81 \pm 0.26(\mathrm{P}=0.013)$. The a value changed significantly from $4.27^{\circ} \pm 1.64^{\circ}$ to $0.15^{\circ} \pm 5.68^{\circ}(\mathrm{P}=0.006)$. This finding shows that the external soft tissue contour was elevated through the bone grafting (Fig. 4).A 3D facial CT analysis showed significant elevation of the pyriform aperture $(\mathrm{n})$ from $0.67 \pm 0.35$ $\mathrm{cm}$ to $0.21 \pm 0.25 \mathrm{~cm}(\mathrm{P}<0.001)$. The (a) value changed significantly from $22.88^{\circ} \pm 16.32^{\circ}$ to $7.94^{\circ} \pm 8.36^{\circ}(\mathrm{P}<0.001)$ (Table 2) (Fig. 5).

\section{DISCUSSION}

The use of an adequate volume of bone in secondary alveolar bone grafts may help achieve excellent periodontal attachment to the adjacent teeth, restore maxillary continuity, induce dental eruption at the cleft site and aesthetic improvement through gingival recovery, and minimize the interruption of facial growth $[5,6]$. However, an inadequate volume of grafted bone can cause graft failure, while too much grafting and excessive compression can lead to inordinate resorption $[7,8]$. We grafted a slightly excessive amount of graft material to achieve an augmentation

\begin{tabular}{|c|c|c|c|c|}
\hline Value & Preoperative & Postoperative & Difference & P-value ${ }^{a)}$ \\
\hline \multicolumn{5}{|c|}{ Photograph } \\
\hline$n / r$ & $0.00 \pm 0.12$ & $0.09 \pm 0.13$ & $-0.10 \pm 0.11$ & $0.005^{c)}$ \\
\hline $\mathrm{a}\left({ }^{\circ}\right)$ & $4.27 \pm 1.64$ & $0.15 \pm 5.68$ & $4.12 \pm 4.92$ & $0.006^{c)}$ \\
\hline $\mathrm{p} / \mathrm{r}$ & $0.69 \pm 0.25$ & $0.81 \pm 0.26$ & $-0.13 \pm 0.04$ & $0.013^{\mathrm{b})}$ \\
\hline \multicolumn{5}{|l|}{ 3D CT } \\
\hline $\mathrm{n}(\mathrm{cm})$ & $0.67 \pm 0.35$ & $0.21 \pm 0.25$ & $0.47 \pm 0.27$ & $<0.001^{\mathrm{d})}$ \\
\hline $\mathrm{a}\left({ }^{\circ}\right)$ & $22.88 \pm 16.32$ & $7.94 \pm 8.36$ & $14.94 \pm 3.24$ & $<0.001^{\text {d) }}$ \\
\hline
\end{tabular}

3D CT, three-dimensional computed tomography; $n$, depression length; r, radius of corneal limbus; a, angle of depression; $p$, thickness of the philtrum.

${ }^{a}$ Wilcoxon signed-rank test; ${ }^{b} P<0.05$; ${ }^{\text {cl }} P<0.01$; ${ }^{\text {dl }} P<0.001$.

effect of the nostril base in our patients, and no complications such as graft failure or inordinate resorption were observed.

The shortcoming of this study was that photogrammetric analysis was used rather than anthropometric analysis. Farkas et al. [9] reported that a number of landmarks and measurements could be considered reliable on photographic measurements. However, they highlighted possible sources of error in photographic measurements when comparing anthropometric and photogrammetric measurements. Bearn et al. [10] noted that 
when using photogrammetry to assess the soft tissues in patients with unilateral cleft lip and palate, photographs are reliable and robust under a range of conditions. In particular, Han et al. [11] reported that fully standardized photogrammetry does not differ statistically from anthropometry. Our facility does not have completely standardized clinical photographic equipment. Each patient was seated on a chair placed $0.5 \mathrm{~m}$ in front of a grey background. The face was relaxed completely, and the patient's hair was arranged to expose the ears. The patient was asked to look at a point on the distant horizon in order to achieve a natural head position in which the Frankfurt horizontal was parallel to the floor. A camera (Canon DSRL 500D, Canon Inc., Tokyo, Japan) with a Canon EFS 18 to $55 \mathrm{~mm}$ lens set on $55 \mathrm{~mm}$ was used. A photograph was obtained at a distance of $1 \mathrm{~m}$ from the patient. A worm's-eye view profile was obtained by a single nurse trained in clinical photography. The distances and angles were measured 3 times to minimize intraobserver variance. We were not able to calculate interobserver variance.

In conclusion, alveolar bone grafting not only fills the gap in the alveolar process but also augments the nostril base after surgery. In this study, only an alveolar bone graft was performed, preventing bias induced by other procedures. Surgeons should consider the augmentation effect of secondary alveolar bone grafting to achieve aesthetic results in the soft tissue contour of the nostril base when performing surgery on children. A future study on how the quantity of the graft will affect the nostril base level is needed for predictable results.

\section{REFERENCES}

1. Hoffman WY, Mount D. Cleft palate repair. In: Mathes SJ, Hentz VR, editors. Plastic surgery. 2nd ed. Vol. 4. Philadelphia: Sanders Elsevier; 2006. p.263-6.
2. Bergland O, Semb G, Abyholm FE. Elimination of the residual alveolar cleft by secondary bone grafting and subsequent orthodontic treatment. Cleft Palate J 1986;23:175-205.

3. United Nations Human Rights. 2002 Convention on the Rights of the Child [Internet]. Geneva: Switzerland; c2013 [cited 2013 Aug 17]. Available from: http://www.ohchr. org/en/professionalinterest/pages/crc.aspx.

4. Hoffman WY, Mount D. Cleft palate repair. In: Mathes SJ, Hentz VR, editors. Plastic surgery. 2nd ed. Vol. 1. Philadelphia: Sanders Elsevier; 2006. p.159-161.

5. Lee C, Crepeau RJ, Williams HB, et al. Alveolar cleft bone grafts: results and imprecisions of the dental radiograph. Plast Reconstr Surg 1995;96:1534-8.

6. Kim JR, Jin SJ, Cho YC, et al. Clinical study of autogenous secondary bone grafting in cleft maxilla. J Korean Assoc Maxillofac Plast Reconstr Surg 2001;23:162-8.

7. Stassen LF. Alveolar bone grafting-how I do it. In: Booth PW, Hausamen JE, Schendel SA, editors. Maxillofacial surgery. Edinburgh: Churchill Livingstone; 1999. p.1047-55.

8. Kim KR, Kim S, Baek SH. Change in grafted secondary alveolar bone in patients with UCLP and UCLA: a threedimensional computed tomography study. Angle Orthod 2008;78:631-40.

9. Farkas LG, Bryson W, Klotz J. Is photogrammetry of the face reliable? Plast Reconstr Surg 1980;66:346-55.

10. Bearn DR, Sandy JR, Shaw WC. Photogrammetric assessment of the soft tissue profile in unilateral cleft lip and palate. Cleft Palate Craniofac J 2002;39:597-603.

11. Han K, Kwon HJ, Choi TH, et al. Comparison of anthropometry with photogrammetry based on a standardized clinical photographic technique using a cephalostat and chair. J Craniomaxillofac Surg 2010;38:96-107. 